\title{
PRADER-WILLI SYNDROME
}

The clinical and polysomnographic features of 5 patients with PraderWilli syndrome were studied at the Mayo Clinic, Jacksonville, FL. All patients, including 2 adults, 1 adolescent, and 2 children were obese; they had hypersomnolence and daytime hypoxemia, and they snored. The apnea plus hyponea index was less than 10 episodes per hour of sleep. Polysomnography failed to demonstrate clinically severe obstructive apnea or hypopnea. Sleep architecture and rapid eye movement latency were normal in all patients. Nonapneic reductions in oxyhemoglobin saturation were detected in only 1 adult and in 1 child. Despite the presence of morbid obesity and a history of snoring these patients with Prader-Willi syndrome had only mild sleepdisordered breathing (Kaplan J et al. Sleep and breathing in patients with the Prader-Willi syndrome. Mayo Clin Proc Nov 1991; 66:1124-1126).

COMMENT. Prader-Willi syndrome is characterized by infantile hypotonia, early childhood obesity, mental deficiency, short stature, small hands and feet and hypogonadism. Many patients also have hypersomnolence, daytime hypoventilation, and some die prematurely of cardiorespiratory failure. The epidemiology of Prader-Willi syndrome was studied in a rural county in Sweden and reported from the Department of Pediatrics and Child Psychiatry, Child Neuropsychiatry Centre, Goteborg, Sweden (Akefeldt A et al. Dev Med

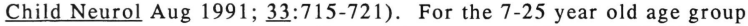
the prevalence of Prader-Willi syndrome was 13 per 100,000 for definite cases, and 20 per 100,000 if borderline cases were included.

Anesthesia and the Prader-Willi syndrome was the subject of a report from the John Radcliffe Hospital, Oxford, England (Mackenzie

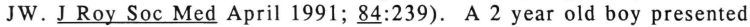
for correction of ventral curvature of micropenis and an 18 year old received anesthetic for removal of excess tissue from one thigh. Halothane in nitrous oxide and oxygen were used for induction and isoflurane to maintain anesthesia. Attention to glycaemic control and body temperature is important since glucose intolerance and both hypothermia and hyperthermia have been reported in response to operative stress.

\section{ACROCALLOSAL SYNDROME}

A large highly inbred kindred with 3 definite and 1 possibly affected siblings with acrocallosal syndrome is reported from the Kaplan Hospital, Rehovot and the Emek Central Hospital, Afula, Israel. The family is of Israeli-Arab extraction and the parents are first cousins. The clinical features are divided into three groups: a) diagnostic/essential findings; b) frequent findings; and c) occasional/associated features. The diagnostic findings include macrocephaly, prominent forehead, hypertelorism, epicanthal folds, downslanted palpebral fissures, small nose with wide nasal bridge, polydactyly and absent/hypoplastic corpus callosum. Frequent findings include ear anomalies, short upper lip, high arched palate, tapered fingers, hypotonia, and 
seizures. Polysyndactyly is the single most variable main sign, both in degree and in presence, even within the same family. A review of 18 published cases and the present family shows that the main manifestations are cranial facial anomalies and the abnormality of the corpus callosum. Most families have been reported from northeastern Switzerland and the Mediterranean basin. The present family supports a chromosomal recessive inheritance (GelmanKohan $\mathrm{Z}$ et al. Further delineation of the acrocallosal syndrome. Eur J Pediatr Sept 1991; 150:797-799).

COMMENT. The acrocallosal syndrome (Schinzel syndrome) and other syndromes which include callosal agenesis are reviewed in Progress in Pediatric Neurology, Ed. Millichap JG, Chicago and London, PNB Publishers 1991, 310-312. At least four distinct syndromes include callosal agenesis as a major component: Aicardi (with infantile spasms, hypsarrythmia, chorioretinal lacunae and coloboma), Schinzel syndrome (with macrocephaly and polysyndactyly), Anderman syndrome (with anterial horn cell disease); and Shapiro syndrome (with recurrent hypothermia).

\section{SEIZURE DISORDERS}

\section{NEURONAL MIGRATION DISORDERS}

Thirty patients with intractable partial epilepsy and a pathological or radiological diagnosis of neuronal migration disorders (NMD) were studied at the Montreal Neurological Institute and Hospital, Montreal, Quebec, Canada. Age at onset of epilepsy ranged from 4 months to 21 years (mean 5.7). Prenatal etiological factors in 8 patients included maternal $x$-ray exposure or trauma, diabetes, advanced maternal age, uterine abnormality. All patients had partial epilepsy: complex partial $(67 \%)$, partial motor $(73 \%)$, and secondary generalized seizures $(73 \%) .8$ patients $(27 \%)$ had tonic or atonic drop attacks, and 6 of these had lesions involving the central region. Status epilepticus had occurred in $30 \%$. A below average IQ was found in $1 / 2$ of the patients. MRI was superior to CT, but did not distinguish between pachygyria, cortical dysplasia or tuberous sclerosis. White matter subcortical abnormalities were demonstrated more frequently than cortical abnormalities. In 22 of 26 surgically treated patients, the histological diagnosis was focal cortical dysplasia (12), and forme fruste of tuberous sclerosis (10). Those with a histological picture of tuberous sclerosis had none of the classical clinical diagnostic signs, but $80 \%$ had delayed psychomotor development and all 10 patients had multilobar unilateral or bilateral EEG spiking. These findings differed from patients with focal cortical dysplasia whose developmental milestones were normal and epileptogenic discharges were confined to one lobe or area of the brain (Palmini A, Andermann F et al. Focal neuronal migration disorders and intractable partial epilepsy: a study of 30 patients. Ann Neurol Dec 1991; $\underline{30: 741-749) \text {. }}$ 